\title{
A Major Upgrade of the H.E.S.S. Cherenkov Cameras
}

Iryna Lypova ${ }^{1, a}$, Gianluca Giavitto ${ }^{1, b}$, Terry Ashton ${ }^{2}$, Arnim Balzer ${ }^{3}$, David Berge ${ }^{3}$, Francois Brun $^{4}$, Thomas Chaminade ${ }^{4}$, Eric Delagnes ${ }^{4}$, Gerard Fontaine ${ }^{6}$, Matthias Füßling ${ }^{1}$, Berrie Giebels $^{6}$, Jean-Francois Glicenstein ${ }^{4}$, Tobias Gräber ${ }^{1}$, Jim Hinton ${ }^{7}$, Albert Jahnke ${ }^{7}$, Stefan Klepser $^{1}$, Marko Kossatz ${ }^{1}$, Axel Kretzschmann ${ }^{1}$, Valentin Lefranc ${ }^{1,5}$, Holger Leich ${ }^{1}$, Hartmut Lüdecke $^{1}$, Pascal Manigot ${ }^{6}$, Vincent Marandon ${ }^{7}$, Emmanuel Moulin ${ }^{4}$, Mathieu de Naurois ${ }^{6}$, Patrick Nayman ${ }^{5}$, Stefan Ohm ${ }^{1}$, Marek Penno $^{1}$, Duncan Ross ${ }^{2}$, David Salek ${ }^{3}$, Markus Schade ${ }^{1}$, Thomas Schwab ${ }^{7}$, Rachel Simoni ${ }^{3}$, Christian Stegmann ${ }^{1}$, Constantin Steppa ${ }^{1}$, Julian Thornhill ${ }^{2}$, and Francois Toussnel ${ }^{5}$

\author{
${ }^{1}$ DESY, D-15738 Zeuthen, Germany \\ ${ }^{2}$ Department of Physics and Astronomy, The University of Leicester, University Road, Leicester, LE1 7RH, \\ United Kingdom \\ ${ }^{3}$ GRAPPA, Anton Pannekoek Institute for Astronomy, University of Amsterdam, Science Park 904, 1098 XH \\ Amsterdam, The Netherlands \\ ${ }^{4}$ DSM/Irfu, CEA Saclay, F-91191 Gif-Sur-Yvette Cedex, France \\ ${ }^{5}$ LPNHE, Université Pierre et Marie Curie Paris 6, Université Denis Diderot Paris 7, CNRS/IN2P3, 4 Place \\ Jussieu, F-75252, Paris Cedex 5, France \\ ${ }^{6}$ Laboratoire Leprince-Ringuet, Ecole Polytechnique, CNRS/IN2P3, F-91128 Palaiseau, France \\ ${ }^{7}$ Max-Planck-Institut für Kernphysik, P.O. Box 103980, D-69029 Heidelberg, Germany
}

\begin{abstract}
The High Energy Stereoscopic System (H.E.S.S.) is an array of imaging atmospheric Cherenkov telescopes (IACTs) located in Namibia. It was built to detect Very High Energy (VHE, $>100 \mathrm{GeV}$ ) cosmic gamma rays, and consists of four $12 \mathrm{~m}$ diameter Cherenkov telescopes (CT1-4), built in 2003, and a larger $28 \mathrm{~m}$ telescope (CT5), built in 2012. The larger mirror surface of CT5 permits to lower the energy threshold of the array down to $30 \mathrm{GeV}$. The cameras of CT1-4 are currently undergoing an extensive upgrade, with the goals of reducing their failure rate, reducing their readout dead time and improving the overall performance of the array. The entire camera electronics has been renewed from ground-up, as well as the power, ventilation and pneumatics systems, and the control and data acquisition software. Technical solutions forseen for the next-generation Cherenkov Telescope Array (CTA) observatory have been introduced, most notably the readout is based on the NECTAr analog memory chip. The camera control subsystems and the control software framework also pursue an innovative design, increasing the camera performance, robustness and flexibility. The CT1 camera has been upgraded in July 2015 and is currently taking data; CT2-4 will upgraded in Fall 2016. Together they will assure continuous operation of H.E.S.S at its full sensitivity until and possibly beyond the advent of CTA. This contribution describes the design, the testing and the in-lab and on-site performance of all components of the newly upgraded H.E.S.S. camera.
\end{abstract}

\footnotetext{
ae-mail: iryna.lypova@desy.de

be-mail: gianluca.giavitto@desy.de
} 


\section{Introduction}

The H.E.S.S. experiment is situated at $1800 \mathrm{~m}$ hight in the Khomas Highland of Namibia. The first four telescopes (CT1 - 4) were built in 2002 - 2003 (H.E.S.S. phase I). They are arranged in a square with $120 \mathrm{~m}$ side length and have $12 \mathrm{~m}$ diameter. In summer 2012 the fifth telescope (CT5) with a diameter of $28 \mathrm{~m}$ was build in the middle of the array (H.E.S.S. phase II). The larger mirror of the new telescope lowered the array energy threshold from $\sim 100 \mathrm{GeV}$ to $\sim 30 \mathrm{GeV}$ and increased the array trigger rate from $(200-300) \mathrm{Hz}$ to more than $1.5 \mathrm{kHz}$. The dead time of the old H.E.S.S. phase I camera was $\sim 450 \mu \mathrm{s}$, which is much bigger than the dead time of the modern CT5 ( $15 \mu \mathrm{s})$ and is not suitable for such high rates. Thus, significant fraction of the triggered events is recorded only by CT5, limiting the performance of the array.

Thereby, one of the main goals of the H.E.S.S. phase I upgrade is to reduce the dead time and to increase the number of recorded stereoscopic events. The second reason for the upgrade is the aging of the electronic components. The cameras of CT1-4 have been operating in the desert environment for more than 10 years leading to an increased rate of failures. To avoid further degradation, the electronics and cabling of the cameras are renewed during the upgrade.

\section{Camera components}

As it is seen from figure 1 most of the electronic components of the camera were replaced. Only photomultiplier tubes (PMTs), their bases, single photoelectron (ph.e.) and flat fielding units for calibration are the same.

The upgraded camera has a new concept for the ventilation system. Instead of many small fans located inside the camera body now there is a single fan on the back door. It increases air pressure and prevents dust accumulation inside the camera. In addition it can heat or cool air if needed. A new back door design was developed for the upgraded ventilation system. However this increased the weight of the back door and required two pneumatic cylinders to open and close it.

Behind the back door there is a rack with back-end electronics. It includes a power distribution box (PDB) and a drawer interface box (DIB). The PDB provides power to the front-end electronics. The DIB is one of the most important components in the camera and is responsible for the interface of the calibration units, ventilation system, number of different sensors, GPS timestamps for the events. Its most important component is the camera trigger.

\subsection{Front-end electronics}

The telescope camera contains 960 PMTs arranged in 60 modules (16 PMTs in each) which are called drawers. A drawer includes two analog boards and one slow control board. Each analog board obtains signals from 8 PMTs. The signals are pre-amplified and sent in tree different channels. One of them is a trigger channel and two others are related to readout (low and high gain).

The trigger channel is fed to a comparator. If the signal is high enough (several photoelectrons) then pixel is triggered (level 0 trigger) [1]. The level 0 trigger signals are routed to the DIB, where $\mathrm{N}$-majority trigger is built out of 38 overlapping groups of 64 pixels each. This is the camera-wide trigger (level 1), which helps to reduce night sky background (NSB) and identify useful data (air showers).

At the same time in readout channels signal gets into NECTAr chip [2] where it is sampled, stored and digitised. There are two capacitor arrays for the signal storage. Each of them has a length of 1024 cells, but if camera is triggered only 16 cells will be read. 


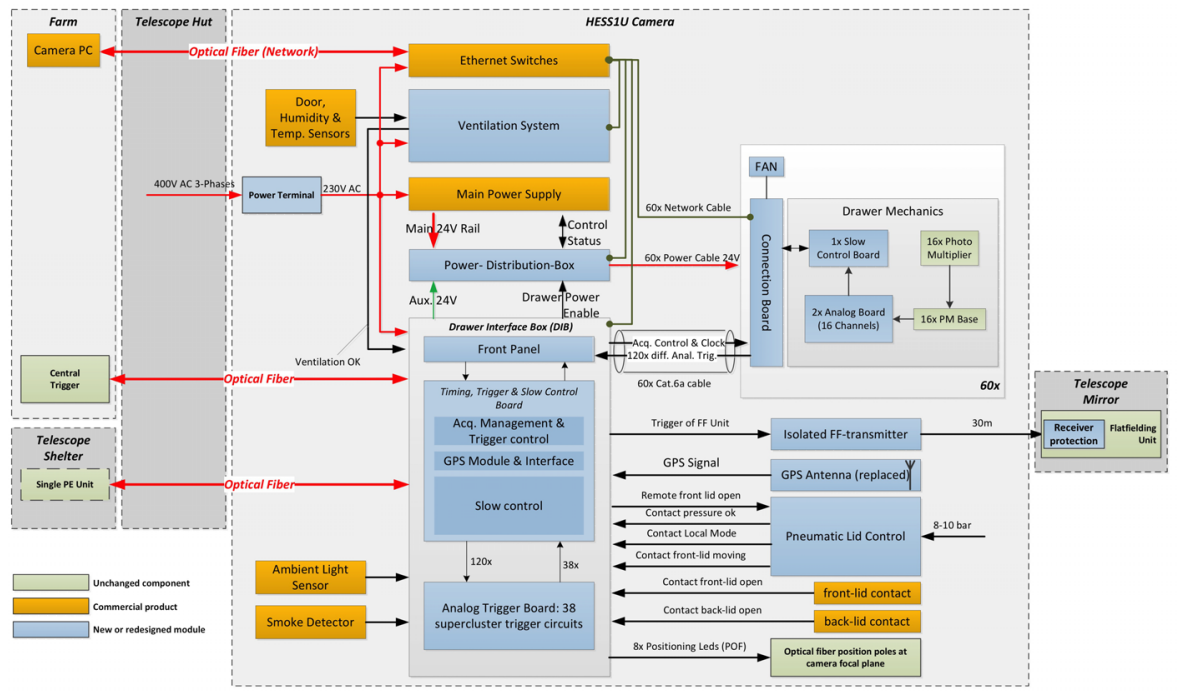

Figure 1: Schematic illustration of upgraded H.E.S.S. camera

\subsection{Tests}

More than 270 drawers were tested at DESY (Zeuthen) with several test setups. A copy of the camera body (CopyCam, fig. 2, left top) was used for tests of the ventilation system, back door mechanics, pneumatics, centre camera trigger etc. The MiniCam is a dark box for four drawers with PMTs, which offered a possibility to check the function of front-end electronics in realistic conditions.

One more test bench (fig. 2, left bottom) was used to check basic functionality of the electronic components and test the drawers without PMTs. Specially for this setup 8-channel pulse generator was developed. It can generate negative pulses with $\sim 1 \mathrm{~ns}$ rising and falling time and amplitudes from $\sim 0.6 \mathrm{mV}$ to $\sim 300 \mathrm{mV}$. A number of different characteristics were tested here: low gain (LG) and high gain (HG) linearity, cross-talk, pedestal noise, trigger path etc. An example of the linearity test results is illustrated on figure 2 (right). High gain shows linear behaviour from 0 to 200 ph.e., low gain from $\sim 30$ ph.e. to 4200 ph.e. Deviation from linearity do not exceed $2 \%$.

\section{Status of the upgrade}

The first camera (CT1) was upgraded in summer 2015 and since March 2016 has been included in regular observation. The upgrade of other three cameras started in September 2016 and currently they are in the process of commissioning. Monte-Carlo simulations for the upgraded cameras are ongoing. For that purpose CORSIKA [3] and sim_telarray [4] are used. First results showed good match between the simulated trigger rate and the observed one with real upgraded camera.

H.E.S.S. phase I upgrade allows us to keep H.E.S.S. operating in a stable regime and improves the sensitivity of the lowest and highest energies. New fast readout electronics gives us the opportunity to lower the trigger threshold and as a result collect more stereoscopic events at energies $<100 \mathrm{GeV}$. In turn, the optimisation of the length of the readout window could improve reconstruction of high energy events. Moreover, the current upgrade of the H.E.S.S. telescopes is a great opportunity to test new electronics, software and algorithms for the future ground based experiments. 

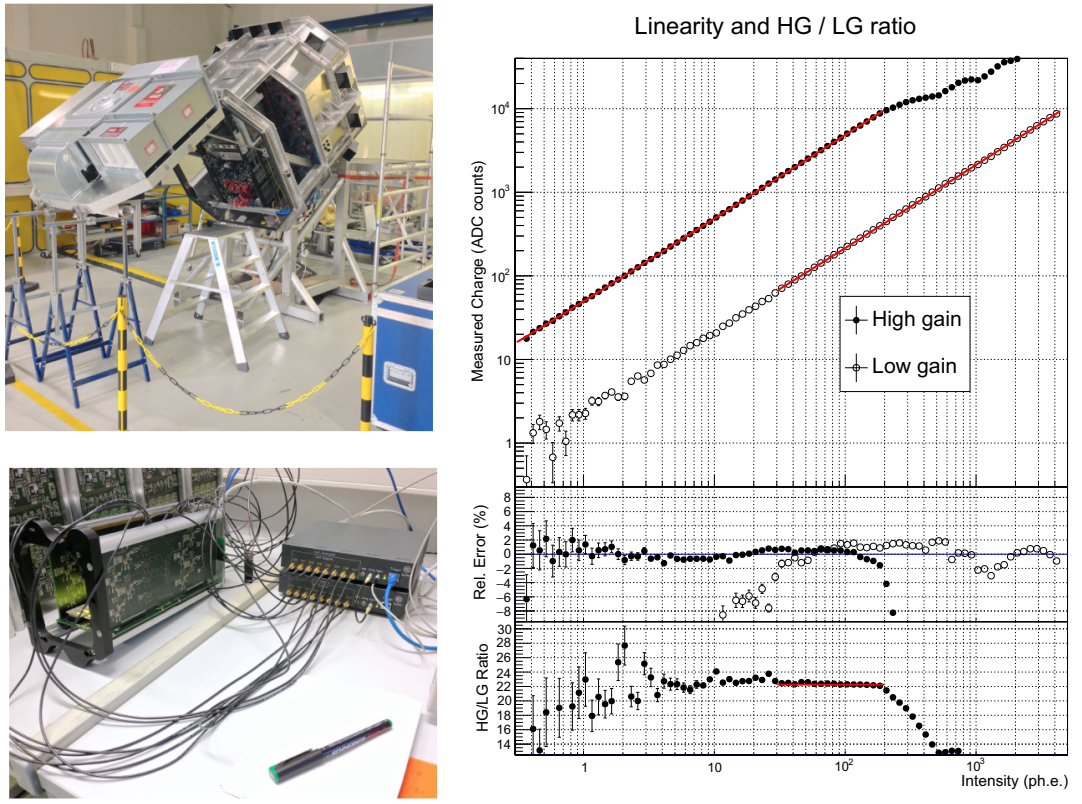

Figure 2: Left: testing setups at DESY (Zeuthen) - CopyCam (top) and test bench with pulse generators (bottom); right: result of linearity tests.

\section{Acknowledgments}

The support of the Namibian authorities and of the University of Namibia in facilitating the construction and operation of H.E.S.S. is gratefully acknowledged, as is the support by the German Ministry for Education and Research (BMBF), the Max Planck Society, the German Research Foundation (DFG), the French Ministry for Research, the CNRS-IN2P3 and the Astroparticle Interdisciplinary Programme of the CNRS, the U.K. Science and Technology Facilities Council (STFC), the IPNP of the Charles University, the Czech Science Foundation, the Polish Ministry of Science and Higher Education, the South African Department of Science and Technology and National Research Foundation, and by the University of Namibia. We appreciate the excellent work of the technical support staff in Berlin, Durham, Hamburg, Heidelberg, Palaiseau, Paris, Saclay, and in Namibia in the construction and operation of the equipment.

\section{References}

[1] Funk, S. et al., Astropart. Phys. 22, 285-296 (2004).

[2] Naumann, C. L. et al., NIM A 695, 44-51 (2012).

[3] Heck D. et al., Wissenschaftliche Berichte FZKA 6019 (1998).

[4] Bernlöhr K., Astropart. Phys. 30, 149-158 (2008). 